\title{
Effect of bench press using maximal and submaximal loads on the Sticking Region
}

\section{Efecto del press de banca utilizando cargas máximas y submáximas en la fase de Sticking Region}

\section{Teresa Valverde-Esteve ${ }^{1}$, Juan Manuel García-Manso ${ }^{2}$}

1 Departamento de Didáctica de la Expresión Musical, Plástica y Corporal. Universitat de València, España.

2 Departamento de Educación Física. Universidad de Las Palmas de Gran Canaria, España.

CORRESPONDENCIA:

Teresa Valverde Esteve

teresa.valverde@uv.es

Recepción: octubre 2020 • Aceptación: abril 2021
CÓMO CITAR EL ARTÍCULO:

Valverde-Esteve, T., \& García-Manso, J.M. (2021). Effect of bench press using maximal and submaximal loads on the Sticking Region. Cultura, Ciencia y Deporte, 16(49), 497-503. http://dx.doi. org/10.12800/ccd.v16i49.1605

\section{Abstract}

The aim of this study was to analyze the Sticking Region at submaximal $(70 \%, 80 \%, 90 \%$ until failure) and maximal (1RM) loads in the concentric phase during bench press. Eleven male individuals participated in the study (age: $22.73 \pm 2.57$ years, body mass: $80.2 \pm 6.9 \mathrm{~kg}$, height: $179.6 \pm 7.4 \mathrm{~cm}, 1 \mathrm{RM}: 94.55 \pm 11.56 \mathrm{~kg}$ ) with at least two years of bench press training experience. In our sample, The Sticking Region could only be observed with $90 \%$ and $100 \% 1 \mathrm{RM}$ loads. The velocity results showed significant differences in 1RM $(p \leq 0.001)$ and in 90\% ( $p=0.014)$ during the three phases (Pre-Sticking, Sticking Region and Post-Sticking zones). Differences in the electromyographic activity of the triceps brachii were found only between the Pre-Sticking Region and the Sticking Region and between the Pre-Sticking Region and the Post-Sticking Region at 1RM. The activity between the Sticking Region and Post-Sticking Region at $90 \%$ and $1 \mathrm{RM}$ did not differ significantly. This study suggests that the Sticking Region is more visible with maximum loads (1RM) due to a weak mechanical position.

Key words: braking phase, chest press, triceps brachii, electromyography.

\section{Resumen}

El objeto de este estudio fue analizar la Sticking Region en las cargas submáximas (70\%, 80\%, 90\% hasta el fallo) y máximas (1RM) durante la fase concéntrica en press de banca. Para este estudio, fueron once los participantes (edad: 22,73 $\pm 2,57$ años; masa corporal: $80,2 \pm 6,9$ kg; altura: 179,6 \pm 7,4 cm; 1RM: $94,55 \pm 11,56 \mathrm{~kg}$ ) con al menos dos años de experiencia en press de banca. En nuestra muestra, la Sticking Region solo pudo ser observada en las cargas del $90 \%$ y $100 \%$ 1RM. Los resultados de velocidad mostraron diferencias estadísticamente significativas en 1RM $(p \leq 0,001)$ y en $90 \%$ ( $p=$ 0,014 ) durante las tres fases (Pre-Sticking, Sticking Region y Post-Sticking). Observamos diferencias en la actividad electromiográfica del tríceps entre las regiones Pre-Sticking y Sticking, así como entre la region Pre-Sticking y Post-Sticking en la carga de $1 \mathrm{RM}$. La actividad entre la Sticking Region y Post-Sticking Region, en las cargas del $90 \%$ y $100 \%$ no mostró diferencias estadísticamente significativas. Este estudio sugiere que la Sticking Region es más visible en cargas máximas (1RM) debido a una baja posición mecánica.

Palabras clave: fase de frenado, press de banca, tríceps braquial, electromiografía. 


\section{Introduction}

Bench press (BP) is one of the most common exercises used for upper body strength training by practitioners of different sports (Borba et al., 2018; Marqués et al., 2007; Drinkwater et al., 2005). BP is especially suitable for increasing the strength of the anterior thorax (pectoralis major and minor muscles), arms (long, medial and lateral portions of the triceps brachii muscles) and shoulders (medial and anterior deltoid muscles) (Barnett et al., 1995; Saeterbakken et al., 2011; Van den Tillaar \& Ettema, 2013). For this reason, numerous studies have focused on the kinematic analysis of BP (Saeterbakken et al., 2011; Van den Tillaar \& Ettema, 2013; 2010; Van den Tillaar \& Saeterbakken, 2013).

By analyzing the force-time curve, it was found that when working with near maximum intensities (e.g. 1RM BP testing) there is a moment during the ascendant phase in which the bar decelerates or even stops before accelerating again (Madsen and McLaughlin, 1984). This region or phase is called the Sticking Region (Lander et al., 1985; Elliott et al., 1989) or Sticking Period (Van den Tillaar \& Ettema, 2010). It is important to note that during BP this moment does not necessarily determine the end of the movement, but it is a zone in which the applied force decreases from the maximal force levels and load (Frost et al., 2010) (Figure 1).
The causes underlying this behavior are not well understood. Some authors have hypothesized that the existence of this phase is due to a weak technique domain (Elliott et al., 1989; Frost et al., 2010; McLaughlin et al., 1984). Studies (Van den Tillaar \& Ettema, 2013; 2010) have analyzed triceps activation in the Pre-Sticking, Sticking Region and PostSticking phases of the velocity curve corresponding to maximal velocity 1 , minimal velocity and maximal velocity2, respectively. Elliott et al. (1989) reported the occurrence of a Sticking Region even with submaximal loads in trained athletes when repetitions are performed until concentric failure. Newton et al. (1997) described this region in loads under 90\% 1RM. Frost et al. (2010) observed this phase using 100\% 1RM.

Some studies (Elliott et al., 1989; Newton et al., 1997; Van den Tillaar \& Ettema, 2009) have related the Sticking Region to the elastic deformation force that is generated during the descendent movement of the bar, therefore only being used in the initial part of the concentric phase. Other works suggest that the BP involves a pushing movement during the concentric phase in which there is a situation of mechanical disadvantage that has a negative influence on the muscle structures involved in the movement (Madsen \& Mclaughlin, 1984). In this regard, Madsen and Mclaughlin (1984) stated that the region in which mechanical efficiency decreases is related to the length of the muscles involved in the movement.
Figure 1. Shows the force-time curves in the loads of $30 \%, 40 \%$ $50 \%$ and $70 \% 1$ RM (García-
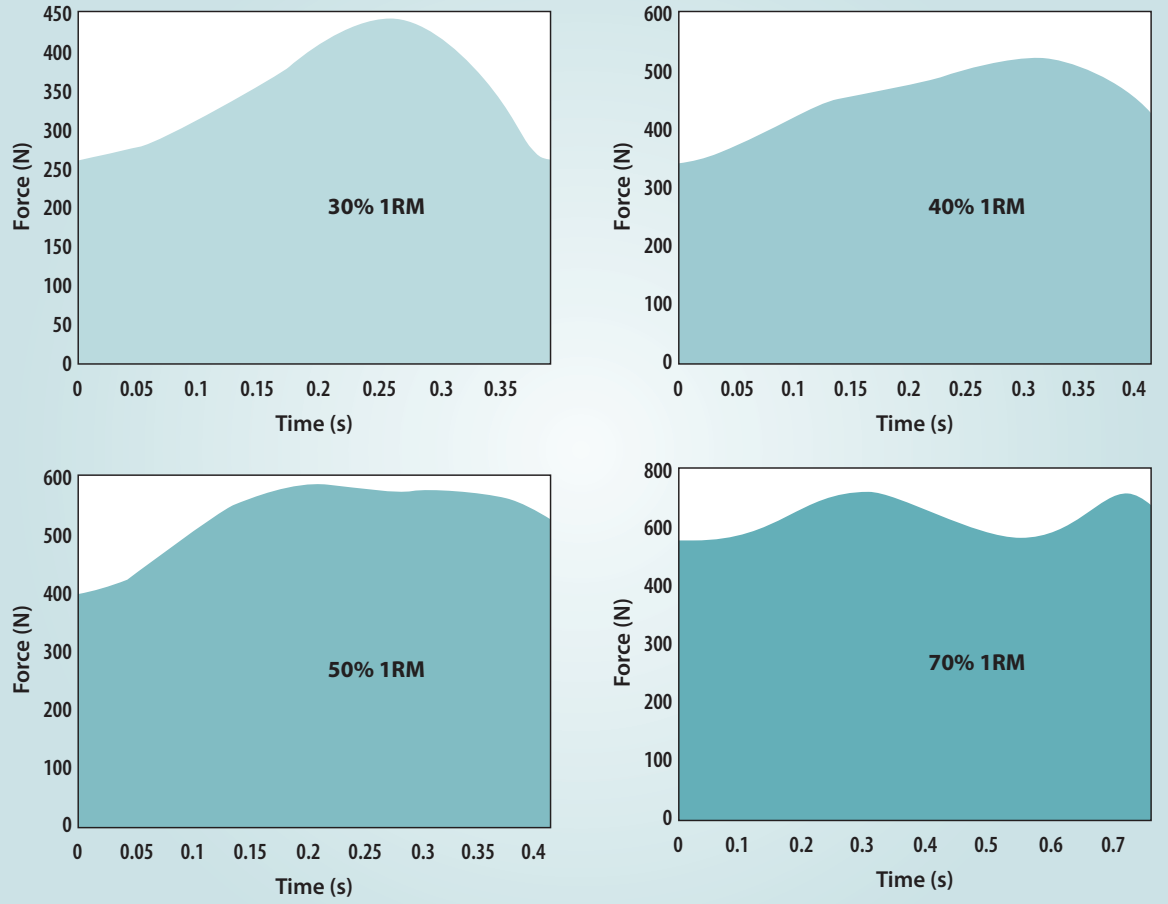
Another possibility could be linked to the width of the grip (Larsen et al., 2021; Lockie et al., 2017; Gomo \& Van Den Tillaar, 2016; Wagner et al., 1992), although it would only occur in the press in which the hands are positioned closer together.

Therefore, the aim of this study was to analyze and compare velocity and bar position during the Sticking Region in repetitions to failure in the loads of $70 \%, 80 \%, 90 \%$ and $100 \% 1 \mathrm{RM}$. Thus, activation of the long portion of the triceps brachii during the concentric phase of the movement at these loads and in the different regions of the movement (Pre-Sticking, Sticking Region and Post-Sticking) was analyzed. The present study will provide coaches and athletes with useful insight into the technique in order to seek strategies to optimize athletes' performance.

The first hypothesis was that the Sticking Region would occur at submaximal and maximal loads (80\%, $90 \%$ and $1 \mathrm{RM}$ ) and at lower displacement in 1RM compared to $90 \%$ and $80 \%$ 1RM. Secondly, maximal velocities and higher triceps brachii activation would occur in the Post-Sticking Region with the loads of $80 \%$, $90 \%$ and 1 RM, showing differences between regions. This study provides a greater understanding of the movement and these phases can be considered when we modify the load and reach maximal intensities.

\section{Methods}

Study design. All the participants came to the laboratory on three different occasions to perform the assessment tests. On the first day, all the participants were informed about the study and carried out the familiarization procedure. In this procedure, participants were instructed to perform movements at $60 \%$ of their body mass, under the indications and corrections of a professional trainer who worked in the laboratory. On the second day, we performed the indirect determination of $1 \mathrm{RM}$ and its subsequent validation. On the third day, all participants performed the repetitions with loads of $90 \%$ (3.09 $\pm 1.30 \mathrm{reps})$, $80 \%$ (7.09 \pm 2.87 reps) and 70\% (12.1 \pm 3.7 reps) $1 \mathrm{RM}$ in a random order until failure as fast as possible. Each participant had a passive rest of 15 minutes after each set of repetitions. During this time, participants did not perform any physical activity that could affect their performance. The width of the hands was determined by the $90^{\circ}$ angle formed by the participants' elbow flexion (105-110 cm distance between hands). The descending bar movement was conducted to the chest. After a 1-second pause, all the participants performed the ascendant part of the movement as fast as possible. This time was controlled using an analogue metronome. Two spotters were standing at either end of the bar for safety reasons and also to encourage the participants. A linear position transducer was placed at the right end of the bar.

Participants. Eleven healthy male participants (age: $22.73 \pm 2.57$ years, body mass: $80.2 \pm 6.9 \mathrm{~kg}$, height: $179.6 \pm 7.4 \mathrm{~cm}, 1 \mathrm{RM}: 94.55 \pm 11.56 \mathrm{~kg}$ ) with at least two years of BP training experience participated in this study. None of the participants performed any body training activity during the 72 hours prior to the assessments. All the participants signed a written informed consent form and all researchers met the guidelines established by the Helsinki Declaration of Human Rights (1965). None of the participants used any drugs or stimulants.

Warm-up. The warm-up protocol consisted of performing 10 repetitions at 40\%, 5 repetitions at 50\% and 4 repetitions at $70 \% 1 \mathrm{RM}$. All repetitions were separated by a 4-minute passive rest. The last warmup set prior to performance was spaced by an 8-minute passive rest interval. These specific warm-ups were conducted after a 10-minute free non-specific warmup in which the participants performed their usual routines.

Determination of 1RM. Prior to determining the load for performing the test at different intensities, 1RM was determined indirectly after performing repetitions with a load of $60 \%$ of the participants' body mass, and calculated (\%load $=1.0278-2.78 \cdot$ No. of Repetitions) (Brzycki, 1993). Once we obtained the result of the calculations, after performing 4 to 6 repetitions, all the participants were given an 8-minute passive rest interval and, finally, the expected 1RM was directly validated through its performance.

Determination of the Sticking Region. Based on previous research (Van den Tillaar \& Saeterbakken, 2013; Van den Tillaar \& Ettema, 2013; Van den Tillaar et al., 2014), we considered three phases regarding the Sticking Region. The first, Pre-Sticking Region, is considered from the start of the concentric phase, when velocity is equal to zero, to the first peak velocity. The second, Sticking Region, runs from the bar peak velocity to the lowest velocity, which is the region at which the velocity is near to zero, following the studies by Van den Tillaar \& Ettema (2013) and Van den Tillaar et al. (2014). In well trained participants with similar intensities, these studies have reported a duration of $\sim 0.50 \mathrm{~s}$ for the Sticking Region and $\sim 1.0$ $\mathrm{s}$ for the Post-Sticking Region. The third, Post-Sticking Region, is considered from the lowest velocity to the second peak velocity. The Sticking Region was only observed in the $90 \%$ and 1 RM loads. 
Triceps brachii rationale. The triceps brachii muscle is responsible for elbow extension. The triceps brachii appears to have high activity due to its size and ability to produce force, and it is also the one whose activity changes the most during BP (Stastny et al., 2017).

Materials. The different BP studies were assessed using an Olympic Salter bar (20 kg; $2.13 \mathrm{~m} ; 2.54 \mathrm{~cm}$ ), bench (Salter, Barcelona, Spain) and 25, 20, 15, 10, 5, 2.5 and $1.25 \mathrm{~kg}$ plates (Salter, Barcelona, Spain). The analysis of the parameters studied (displacement, velocity, force, electromyography) was performed using MuscleLab System Hardware (TM model 4000e, Ergo test Technology, Langesund, Norway) with its corresponding Software (MuscleLab, version 7.18, Ergotest technology A. S, Bosco System. MuscleLab, TM model 4020e). This software synchronizes the parameters of time, acceleration, vertical displacement and electromyography (EMG). Then, it provides the force, and velocity results by multiplying the manual inserted load by the acceleration and displacement. The sampling frequency is $100 \mathrm{~Hz}$ and the measurement resolution $<0.075 \mathrm{~mm}$. Its maximal range is $3.5 \mathrm{~m}$ and the maximal velocity recorded is $24 \mathrm{~m} / \mathrm{s}$. Its dimensions are $135 \times 80 \times 55 \mathrm{~mm}$ and its mass is 550 g. These data are exported to Excel (Microsoft) and can be visualized as graphs.

Electromyography. The EMG signal of the triceps activation was recorded on the right arm of each participant, as all participants were right-handed. Before the 1RM and $90 \%$ experimental tests, the three electrodes (5 cm round pregelled $\mathrm{Al} / \mathrm{AgCl}$ electrodes; Lessa, Barcelona, Spain) were placed on the prepared skin (shaved, washed with alcohol and abraded). The measurement was performed on the long portion of the triceps brachii, following the protocol by Delagi et al. (1981), aligned with the approximate muscle fiber direction and placed in pairs with a $20-\mathrm{mm}$ distance between centers (Cram et al., 1988). All the electrodes were applied by the same person.

Any noise in the EMG signal reported by this system was eliminated using a Butterworth fourth-order filter, with a low cut-off frequency of $8 \mathrm{~Hz}$ and a high frequency of $600 \mathrm{~Hz}$. Once filtered and transformed into the Root Mean Square ( $R M S)$, it reported a sample frequency of $100 \mathrm{~Hz}$.

The parameter assessed was the area of the EMG in the three zones of the movement: Pre-Sticking, Sticking Region and Post-Sticking. Following a similar study (Van den Tillaar \& Ettema, 2013), Pre-Sticking was considered the first acceleration phase, the Sticking Region was the first deceleration phase and Post-Sticking was the second acceleration phase.
Data processing and statistical analysis. From all the parameters, descriptive statistics such as the mean, standard deviation and maximum and minimum values were obtained. After the Shapiro-Wilk normality test, we performed the repeated measures analysis (twoway ANOVA) for comparison of the three zones (PreSticking, Sticking Region and Post-Sticking) and the two intensities at which the Sticking Region was observed (90\% and 1RM), using the Bonferroni procedure for post-hoc analysis. The $70 \%$ and $80 \%$ loads were not evaluated because the regions could not be defined. We also calculated the effect size with $\eta^{2}$ (Eta partial squared). The paired Student's t-test was used to compare the two sets (1RM vs. 90\%). In both tests, the result was significant when $p \leq 0.05$ at a $95 \%$ IC. Also, The EMG signal was exported and processed with external software (MATLAB Statistics Toolbox, MathWorks, USA).

\section{Results}

Our first result, after data analysis, was that the Sticking Region was only observed in the $90 \%$ and 1 RM loads. The force values were higher in 1RM than in 90\% (Figure 2). Specifically, we observed significant differences between the Sticking Region $(\mathrm{t}=3.155, p$ $=0.009)$ and Post-Sticking $(\mathrm{t}=3.294, p=0.007)$ when we compared the $1 \mathrm{RM}$ and $90 \%$ loads. There were significant differences in force in 1RM during the PreSticking, Sticking Region and Post-Sticking phases $\left(\mathrm{F}_{2,33}\right)$ $=16.494, p \leq 0.001, \eta^{2}=0.50$.

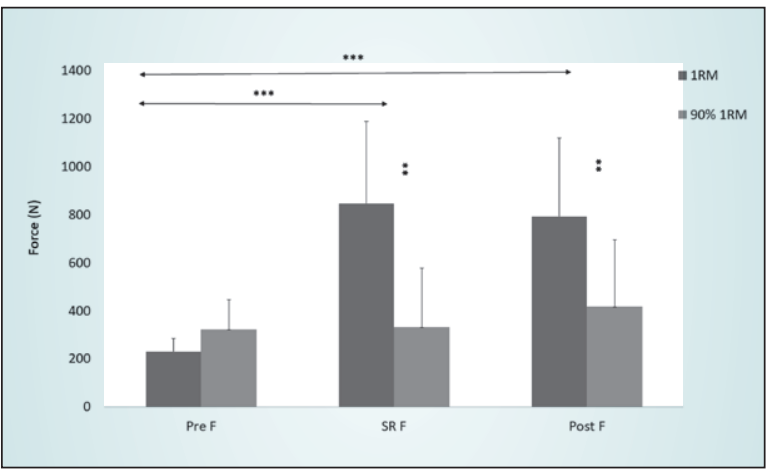

Figure 2. Shows the magnitudes of force in each phase of the force-time curve (Pre-Sticking, Sticking Region and Post-Sticking) in the 1RM and $90 \%$ loads and the significant values between phases and intensities. Note: $* *: \leq 0.010$ between intensities; $* * *: p \leq 0.001$ in the $1 \mathrm{RM}$ load.

Significant differences between loads were only found in the Pre-Sticking zone ( $\mathrm{t}=-2.575, p=0.026)$ in velocity. Velocity was higher in the $90 \%$ load during the Pre-Sticking and Post-Sticking phases, while the highest values were observed in 1RM (Figure 3). 


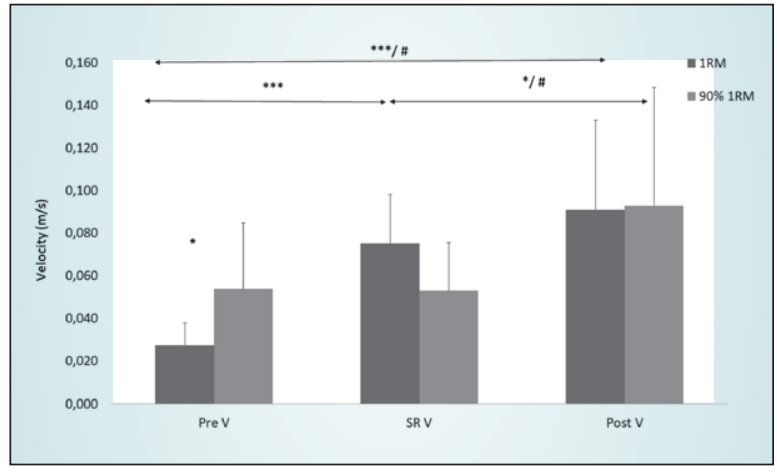

Figure 3. Shows the magnitudes of velocity in each phase of the forcetime curve (Pre-Sticking, Sticking Region and Post-Sticking) in the 1RM and $90 \%$ loads and the significant values between phases and intensities. Note: $* * *: p \leq 0.001$ in the 1 RM load; $\#: p \leq 0.05$ in the $90 \%$ load; $*: p \leq$ 0.05 between intensities.

Values showed significant differences in 1RM during all three phases $\left(\mathrm{F}_{2,33}=15.244 ; p \leq 0.001, \eta^{2}=0.47\right)$. Significant differences were also reported at $90 \%\left(\mathrm{~F}_{2,33}\right.$ $\left.=4.890, p=0.014, \eta^{2}=0.29\right)$.

The Pre-Sticking, Sticking Region and Post-Sticking zones were located earlier in the displacement in the 1RM load than in 90\% (Pre-Sticking ${ }_{90 \%}: 25.35 \pm 13.34 \%$, Pre-Sticking ${ }_{1 \mathrm{RM}}$ : $13.10 \pm 9.10 \%$; Sticking Region ${ }_{90 \%}$ : $42.23 \pm 14.21 \%$; Sticking Region ${ }_{1 \mathrm{RM}}$ : $42.15 \pm 12.17 \%$; Post-Sticking ${ }_{90 \%}: 86.40 \pm 5.55 \%$; Post-Sticking ${ }_{1 \mathrm{RM}}: 84.43$ $\pm 5.32 \%)$. The differences between the displacement in the three phases were also significant in the loads of $1 \mathrm{RM}$ and $90 \%\left(\mathrm{~F}_{2,33}=55.460, p \leq 0.001, \eta^{2}=0.77\right.$ (Figure 4).

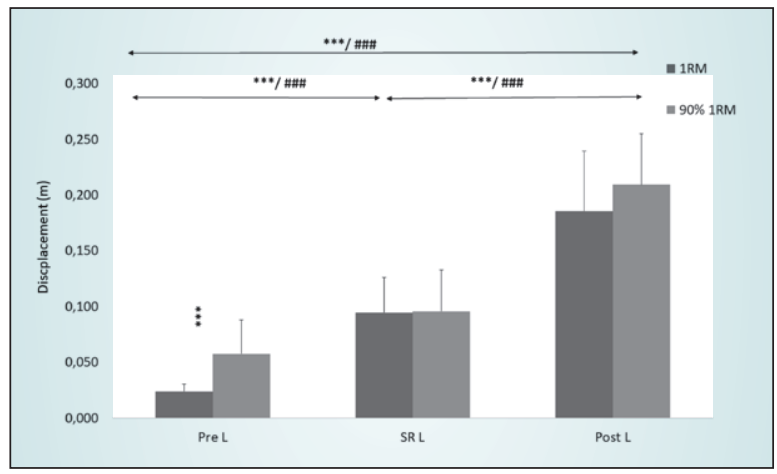

Figure 4. Shows the point of the displacement at which we observed the Pre-Sticking, Sticking Region and Post-Sticking zones in the 1RM and $90 \%$ loads. Note: $* * *: p \leq 0.001$ in the 1RM load; \#\#\#: $p \leq 0.001$ in the $90 \%$ load; $* * *: p \leq 0.001$ between intensities.

The $I_{E M G}$ values did not show significant differences for the two loads. However, this activity was higher in the Pre-Sticking zone at $90 \%$ when compared with 1RM (Figure 4). $I_{E M G}$ only showed significant differences in the 1RM load $\left(\mathrm{F}_{2,33}=18.790, p \leq 0.001, \eta^{2}=0.53\right.$ (Figure 5). The $I_{E M G}$ was higher at $1 \mathrm{RM}$ in the Sticking Region and Post Sticking zones.

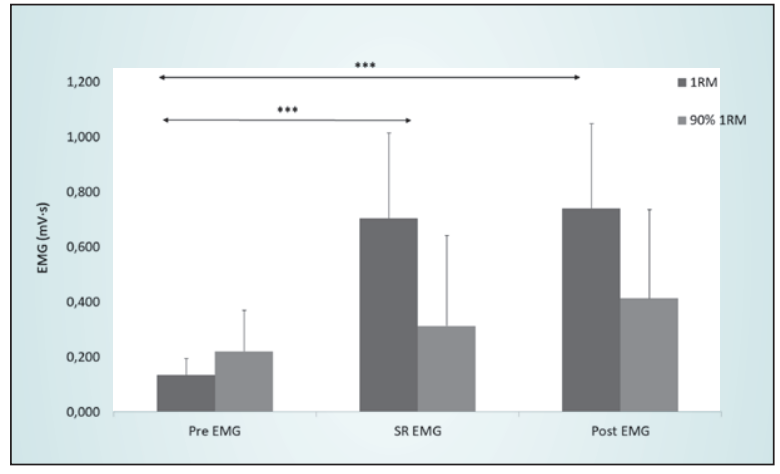

Figure 5. Shows the $I_{E M G}$ activity of the long portion of the triceps brachii at $1 \mathrm{RM}$ and $90 \%$ in every phase of the curve (Pre-Sticking, SR and PostSticking). Note: $* * *: p \leq 0.001$ in the 1 RM load; \#: $p \leq 0.05 ; *: p \leq 0.05$ between intensities.

\section{Discussion}

The aim of this study was to find and compare the Sticking Region in the loads of $70 \%, 80 \%$, 90\% and 1RM. The results of our study showed that this region was only found in the $90 \%$ and 1 RM loads. For this reason, we also examined whether changes in the work load (90\% and 1RM) were reflected in the velocity, displacement and $I_{E M G}$ during BP at submaximal and maximal loads ( $90 \%$ and $1 \mathrm{RM}$ ).

The highest velocity magnitudes were located in the Post-Sticking phase. Consequently, the lowest bar velocities were located in the Sticking Region using $90 \%$ loads. This tendency is very similar to that found in a study comparing concentric BP with countermovement BP, in which no isometric portion was performed before the upward movement (Van den Tillaar \& Ettema, 2013). Specifically, Van den Tillaar et al. (2012) reported the peak velocities in higher magnitudes than in our study $\left(1^{\text {st }}\right.$ highest peak: $\sim 0.26$ $\mathrm{m} / \mathrm{s}$ vs $\sim 0.14 \mathrm{~m} / \mathrm{s}$; lowest peak: $\sim 0.07 \mathrm{~m} / \mathrm{s}$ vs $0.06 \mathrm{~m} / \mathrm{s}$; $2^{\text {nd }}$ highest peak: $\sim 0.35 \mathrm{~m} / \mathrm{s} v s \sim 0.17 \mathrm{~m} / \mathrm{s}$ ), probably due to the characteristics of the sample.

When we observed the displacement in both loads, the values reported in the study by Van den Tillaar et al. (2012) were similar for the Pre-Sticking and Sticking Region zones (Pre-Sticking $\sim 3 \mathrm{~cm}$, Sticking Region $\sim 13 \mathrm{~cm}$, Post-Sticking $\sim 31 \mathrm{~cm}$ ). In the study by Martínez-Cava et al. (2019) the Pre-Sticking was observed $\sim 5.5 \mathrm{~cm}(12.7 \%)$, Sticking Region at $\sim 16 \mathrm{~cm}$ (35.5\%) and Post-Sticking $38.7 \mathrm{~cm}$ (89.4\%), while in our study these peaks were observed at lower values in 1RM (Pre-Sticking $2 \mathrm{~cm}$, Sticking Region $\sim 9 \mathrm{~cm}$, Post-Sticking $\sim 19 \mathrm{~cm}$ ) than in 90\% (Pre-Sticking $6 \mathrm{~cm}$, Sticking Region $\sim 10 \mathrm{~cm}$, Post-Sticking $\sim 21 \mathrm{~cm})$. In both cases, these values correspond to $\sim 12 \%$ for the PreSticking, 42\% Sticking Region and 84\% Post-Sticking 
zones. These differences could be due to the quality of performance and the anthropometric characterization of the sample.

Our first hypothesis on finding the Sticking Region was not entirely confirmed, as this region was only observed in the $90 \%$ and 1RM loads. Also, for both loads, higher peak velocity values were located very close together, at higher vertical displacement. In relative values, the Sticking Region at the $90 \%$ load was located at $42.23 \%$ of displacement and the Sticking Region at $1 \mathrm{RM}$ at $42.15 \%$. These results were very similar to the $35-45 \%$ suggested by Newton et al. (1997) at the $90 \%$ load.

Our second hypothesis was confirmed, as activation of the long portion of the triceps brachii was higher in the Sticking Region and Post-Sticking zones, as suggested by Van den Tillaar and Ettema (2013). To our understanding, the muscle works under better conditions, as there is a relationship between force and muscle length.

Van den Tillaar, et al. (2012) also put forward some hypotheses about the Sticking Region phase. One of their suggestions is that it may be due to the elastic activation (with a retard of $300 \mathrm{~ms}$ ) and neural alterations linked to high loads, and also to diminished potentiation (Van den Tillaar et al., 2012). However, these authors insisted that none of the answers is solid enough to draw any conclusions. From our point of view, in the context of muscle coordination, we consider the possibility, not assessed in this study, of relaxation of the activity of the pectoralis minor or a loss of mechanical efficiency due to the descendent phase, especially in high loads. The triceps brachii is a muscle that originates from the coracoid process of the scapula and during BP its task is to set the shoulder or project it forward.

Under this circumstance, the magnitudes of force can decrease at the moment the pectoral tension cedes. Therefore, Van den Tillaar and Ettema (2009) suggested that the different Pre-Sticking, Sticking Region and Post-Sticking phases occurred due to weak activity of the pectoral and deltoid muscles, probably caused by the braking phase at the end of the movement (Newton et al., 1996). It should be noted that in our study, we assessed the concentric BP after a brief pause at the end of the eccentric phase. For this reason, there was no elastic-reflex component influencing the movement. In this regard, a study by Van den Tillaar and Kwan (2020) reported no significant differences in the Sticking Region in terms of triceps activation when an eccentric component was added to the BP in loads of $85 \%$ and 95\% 1 RM. In future studies, it would be interesting to know if the Sticking Region is obtained during BP when the body position is modified (incline or decline BP). Therefore, further research could be carried out to determine whether the Sticking Region is obtained in the same range of motion and whether the behavior of other muscles is altered when the position of the body is modified.

\section{Conclusions}

In conclusion, the Sticking Region is observed with loads of $90 \%$ and $100 \%$ 1RM. Significant differences were observed in velocity and triceps brachii muscle response in the Sticking Region and Post-Sticking zones when comparing the two loads. This study suggests that the Sticking Region is more visible with maximum loads (1RM) due to a weak mechanical position. Further investigation is needed to compare muscle activity during maximum load lifting.

All authors declare that there is no conflict of interests. 


\section{REFERENCES}

Barnett, C., Kippers, V., Van den Tillaar, R., \& Saeterbakken, A. (2014). Effect of Fatigue Upon Performance and Electromyographic Activity in 6-RM Bench Press. Journal of Human Kinetics, 40(1), 57-65. https:// doi.org/10.2478/hukin-2014-0007

Borba, D. D. A., Batista Ferreira-Júnior, J., Ramos, M. V. D., Gomes, R. D. L. D., Guimarães, J. B., \& Oliveira, J. R. V. D. (2018). Bench press exercise performed as conditioning activity improves shot put performance in untrained subjects. Motriz: Revista de Educação Física, 24(4). DOI: http://doi.org/10.1590/S1980-6574201800040003

Brzycki, M. (1993). Strength testing-predicting a one-rep max from reps-to-fatigue. Journal of Physical Education, Recreation and Dance, 64(1), 88-90.

Cram, J., Kasman, G., \& Holtz, J. (1998). Electrode placements. Introduction to surface electromyography. Gaitersburg: Aspen.

Delagi, E. F., Perotto, A., Lazzetti, J., \& Morrison, D. (1981). Anatomic Guide for the Eletromyographer: the limbs. (2nd edition). Springfield, Illinois: Charles C Thomas Publisher.

Drinkwater, E. J., Lawton, T. W., Lindsell, R. P., Pyne, D. B., Hunt, P. H., \& Mckenna, M. J. (2005). Training leading to repetition failure enhances bench press strength gains in elite junior athletes. The Journal of Strength \& Conditioning Research, 19(2), 382-388.

Elliott, B.C., Wilson, G.J., \& Kerr, G.K. (1989). A biomechanical analysis of the sticking region in the bench press. Medicine and science in sports and exercise, 21(4), 450-462.

Frost, D.M., Cronin, J., \& Newton, R.U. (2010). A biomechanical evaluation of resistance. Sports Medicine, 40(4), 303-326. https://doi. org/0112-1642/10/0004-0303

García-Manso, J.M. \& Valverde-Esteve, T. (2015). Potencia muscular y rendimiento deportivo. Colombia: Kinesis.

Gomo, O., \& Van Den Tillaar, R. (2016). The effects of grip width on sticking region in bench press. Journal of sports sciences, 34(3), 232-238. https://doi.org/10.1080/02640414.2015.1046395

Lander, J.E., Bates, B.T., Sawhill, J.A., \& Hamill, J. (1985). A comparison between free-weight and isokinetic bench pressing. Medicine and science in sports and exercise, 17(3), 344-353.

Larsen, S., Gomo, O., \& van den Tillaar, R. (2021). A biomechanical analysis of wide, medium, and narrow grip width effects on kinematics, horizontal kinetics, and muscle activity on the sticking region in recreationally trained males during 1-RM bench pressing. Frontiers in Sports and Active Living, 2, 229. https://doi.org/10.3389/fspor.2020.637066

Lockie, R. G., Callaghan, S. J., Moreno, M. R., Risso, F. G., Liu, T. M., Stage, A. A., ... \& Orjalo, A. J. (2017). An investigation of the mechanics and sticking region of a one-repetition maximum close-grip bench press versus the traditional bench press. Sports, 5(3), 46. https://doi. org/10.3390/sports5030046

Madsen, N., \& McLaughlin, T. (1984). Kinematic factors influencing performance and injury risk in the bench press exercise. Medicine and science in sports and exercise, 16(4), 376-381.

Martínez-Cava, A., Morán-Navarro, R., Hernández-Belmonte, A., Courel-Ibáñez, J., Conesa-Ros, E., González-Badillo, J. J., \& Pallarés, J. G. (2019). Range of motion and sticking region effects on the bench press load-velocity relationship. Journal of sports science \& medicine, 18(4), 645.
Marques, M. C., Van Den Tillaar, R., Vescovi, J. D., \& González-Badillo, J. J. (2007). Relationship between throwing velocity, muscle power, and bar velocity during bench press in elite handball players. International journal of sports physiology and performance, 2(4), 414-422. https:// doi.org/10.1123/ijspp.2.4.414

McLaughlin, T.M., \& Madsen, N.H. (1984). Bench Press: Bench press techniques of elite heavyweight powerlifters. Strength and Conditioning Journal, 6(4), 44-44.

Newton, R.U., Kraemer, W.J., Haekkinen, K., Humphries, B., \& Murphy, A.J. (1996). Kinematics, kinetics, and muscle activation during explosive upper body movements. Journal of Applied Biomechanics, 12(1), 31-43.

Newton, R.U., Murphy, A.J., Humphries, B.J., Wilson, G.J., Kraemer, W.J., \& Häkkinen, K. (1997). Influence of load and stretch shortening cycle on the kinematics, kinetics and muscle activation that occurs during explosive upper-body movements. European journal of applied physiology and occupational physiology, 75(4), 333-342.

Saeterbakken, A.H., Van den Tillaar, R., \& Fimland, M.S. (2011). A comparison of muscle activity and 1-RM strength of three chest-press exercises with different stability requirements. Journal of sports sciences, 29(5), 533-538. https://doi.org/10.1080/02640414.2010.543916

Stastny, P., Gołaś, A., Blazek, D., Maszczyk, A., Wilk, M., Pietraszewski, P., ... \& Zając, A. (2017). A systematic review of surface electromyography analyses of the bench press movement task. PloS one, 12(2), e0171632. https://doi.org/10.1371/journal.pone.0171632

Van den Tillaar, R., Andersen, V., \& Saeterbakken, A. H. (2014). The existence of a sticking region in free weight squats. Journal of human kinetics, 42(1), 63-71. https://doi.org/10.2478/hukin-2014-0061

Van Den Tillaar, \& Ettema (2009). A comparison of successful and unsuccessful attempts in maximal bench pressing. Medicine and science in sports and exercise, 41(11), 2056-2063. https://oi.org/10.1249/ MSS.0b013e3181a8c360

Van den Tillaar, R., \& Ettema, G. (2010). The "sticking period" in a maximum bench press. Journal of sports sciences, 28(5), 529-535. https:// doi.org/10.1080/02640411003628022

Van den Tillaar, R., \& Ettema, G. (2013). A comparison of muscle activity in concentric and counter movement maximum bench press. Journal of human kinetics, 38, 63-71. https://doi.org/10.2478/hukin-2013-0046

Van den Tillaar, R., \& Kwan, K. (2020). The Effects of Augmented Eccentric Loading upon Kinematics and Muscle Activation in Bench Press Performance. Journal of Functional Morphology Kinesioly, 5(1), 1-12.

Van den Tillaar, R., Saeterbakken, A., \& Ettema, G. (2012). A comparison of muscle activation between maximum pure concentric and counter movement bench pressing. In ISBS-Conference Proceedings Archive, 1 (1).

Van Den Tillaar, R., \& Saeterbakken, A. H. (2013). Fatigue effects upon sticking region and electromyography in a six-repetition maximum bench press. Journal of sports sciences, 31(16), 1823-1830. https://doi. org/10.1080/02640414.2013.803593

Van den Tillaar, R., Saeterbakken, A.H., \& Ettema, G. (2012). Is the occurrence of the sticking region the result of diminishing potentiation in bench press? Journal of Sports Sciences, 30(6), 591-599. https://doi. org/10.1080/02640414.2012.658844

Wagner, L.L., Evans, S.A., Weir, J.P., Housh, T.J., \& Johnson, G.O. (1992). The effect of grip width on bench press performance. Journal of Applied Biomechanics, 8(1), 1-10. 